\title{
Anesthesia and Neurotoxicity, First Edition
}

\author{
Yuji Morimoto (Editor). Springer, 2017, 167 pages. ISBN 978-4-431-55623-7
}

\author{
Oana Predescu, MD, MSc, FRCPS
}

Received: 19 March 2018/Revised: 5 April 2018/Accepted: 8 April 2018/Published online: 18 April 2018

(C) Canadian Anesthesiologists' Society 2018

The first edition of Anesthesia and Neurotoxicity was published shortly after the United States Food and Drug Administration (FDA) issued (December 2016) a "drug safety communication" warning the public that "general anesthesia and sedation drugs used in children less than three years of age or in pregnant women in their third trimester who were undergoing anesthesia for more than three hours or repeated use of anesthetics may affect the development of children's brains." ${ }^{1}$ This 167-page, multiauthored book, with contributions from both clinicians and basic scientists, is a comprehensive literature review that addresses the widespread concern that anesthesia might be harmful to the central nervous system. It focuses on the alarms raised by the FDA and provides evidence-based answers to questions regarding the potential neurotoxic effects of general anesthesia.

The book, published in an easy-to-read format and on high-quality paper, is structured into two parts for a total of ten chapters. Part I, Neurotoxicity of the Anesthetics for Developing Brain, in its first chapter, summarizes in vivo animal studies that assessed the consequences of long-term exposure to anesthetics on brain development. Various neurotoxic mechanisms that mediate learning disability are considered, including apoptosis, inhibition of long-term potentiation, mitochondrial injury, inhibition of synapse formation and pruning, neuro-inflammation, and granule cell ectopy. A nicely outlined explanatory figure illustrates the sequence of mammalian neuronal development (i.e., while the brain is maturing), emphasizing the potential importance of the timing of exposure to a harmful

O. Predescu, MD, MSc, FRCPS ( $\square)$

McGill University, Montreal, QC, Canada

e-mail: oanadp@mac.com substance. This first chapter concludes that, based on animal studies, a causal inference can be made between volatile or intravenous anesthesia exposure and neurotoxicity - with harmful effects on the central nervous system.

The following two chapters in Part I review translational studies that include retrospective and prospective longitudinal clinical studies that address the association of pediatric patients' exposure to a general anesthetic and the possible adverse neuro-developmental outcomes. These studies are difficult to interpret owing to multiple confounding factors, with Part I concluding that "further data are necessary to make a conclusive statement regarding the risk of exposure in young children."

Part II, Postoperative Delirium and Cognitive Dysfunction, is completely independent from Part I. Its seven chapters guide the reader through the definition, clinical manifestations, and treatment of postoperative delirium as well as postoperative cognitive dysfunction, dementia, and stroke. It emphasizes that these manifestations can be difficult to distinguish because the symptoms may overlap and/or be secondary to multifactorial etiologies. Chapter 8, Prevention and Treatment of Postoperative Delirium and Postoperative Cognitive Dysfunction, is noteworthy by its inclusion of an extensive table summarizing the risk factors linked to postoperative delirium and a clear, comprehensive figure summarizing intraoperative interventions that may help reduce the incidence of postoperative cognitive dysfunction. Chapter 10, Mechanism of Postoperative Delirium and Postoperative Cognitive Dysfunction, also offers an excellent figure. Well incorporated into the text, the figure is a representation of the microglial hypothesis of postoperative cognitive dysfunction. 
Unlike Part I, Part II suffers from considerable overlap of topics and redundancy of the literature being reviewed, including the presentation of identical tables. The last phrase of Chapter 10, which ends the book, states that "Physical exercise is the only way to prevent delirium even in critical care setting. The mechanism applies equally to the postoperative phase." This non-referenced conclusion suggests future research opportunities.

Anesthesia and Neurotoxicity - a unique synthesis of basic science and clinical research -is concerned with the neurotoxic effects of anesthetic drugs. It is a concise, up-todate review of the literature and could be a useful reference for anesthesia care providers who may have to allay their patients' concerns about the potential harmful effects of general anesthesia.
Conflicts of interest None declared.

Editorial responsibility This submission was handled by Dr. Steven Backman. Associate Editor, Canadian Journal of Anesthesia.

\section{Reference}

1. U.S. Food and Drug Administration. FDA Drug Safety Communication: FDA review results in new warnings about using general anesthetics and sedation drugs in young children and pregnant women. Available from https://www.fda.gov/Drugs/ DrugSafety/ucm532356.htm (accessed April 2018). 